\title{
Influence du nombre de corps jaunes sur la libération d'ocytocine lutéale, le transfert du lait alvéolaire dans la citerne et la production laitière chez la brebis
}

\author{
J Labussière, PG Marnet, JF Combaud, \\ $M$ Beaufils, FA de la Chevalerie
}

INRA, laboratoire de la Traite, 65, rue de Saint-Brieuc, 35042 Rennes cedex, France

(Reçu le 15 janvier 1993; accepté le 28 juin 1993)

\begin{abstract}
Résumé - Basée sur les sécrétions lutéales d'ocytocine et de progestérone, l'expérience consiste à comparer la production laitière et la répartition des fractions citernales et alvéolaires de 59 brebis Lacaune entre une $1^{\text {re }}$ période (témoin) où elles sont toutes dépourvues de corps jaune, et une $2^{e}$ période (expérimentale) où, à la suite de différents traitements de superovulation, elles en possèdent $0,1,2,3$ à 6 , ou plus de 6 (groupes A, B, C, D et E composés respectivement de 20, 7, 14, 7 et 11 brebis). Les 2 séries de mesures ont lieu à $15 \mathrm{j}$ d'intervalle; elles indiquent que plus il y a de corps jaune, plus les taux plasmatiques d'ocytocine et de progestérone sont élevés, plus le volume de lait citernal est important et le volume de lait alvéolaire faible et enfin plus la production laitière est abondante. C'est ainsi que les volumes totaux recueillis à la traite du soir qui étaient approximativement les mêmes pour les groupes $A$ et $E$ pendant la période témoin $(388,6 \mathrm{ml}$ et $384,8 \mathrm{ml})$ passent respectivement à $321,7 \mathrm{ml}(-17,2 \%)$ et $413,4 \mathrm{ml}(+7,4 \%)$ pendant la période expérimentale. Entre les brebis n'ayant formé aucun corps jaune et celles qui en ont formé plus de 6 , il se crée donc en 2 semaines une différence de $24,6 \%$. Celle-ci est attribuée essentiellement à l'accroissement de l'ocytocinémie et à l'hyperévacuation du lait alvéolaire qu'elle provoque. Cependant, il n'est pas exclu que l'augmentation conjointe de la progestéronémie stimule également la sécrétion des cellules mammaires.
\end{abstract}

ocytocine / corps jaune / éjection lactée / glactopoièse / brebis

Summary - Influence of the number of corpora lutea on the release of luteal oxytocin, the transfer of milk from the alveolar to cisternal lumen and milk production in the ewe. This experiment was conducted in 59 Lacaune breed ewes in order to compare milk production and milk distribution between alveolar and cisternal storage after superovulation. After a corpora lutea (CL)free control period, the ewes were superovulated by different treatments (experimental period) and 5 classes were differentiated according to the number of corpora lutea observed $(0,1,2,3$ to 6 and $>$ $6 C L$ respectively (group $A(n=20), B(n=7), C(n=14), D(n=7), E(n=11))$. Our results showed a positive correlation between the number of corpora lutea and the oxytocin and progesterone levels in plasma, total milk production and cisternal volume, and a negative correlation with alveolar volume. The milk production at the evening milking for groups $A$ and $E(388.6$ and $384.8 \mathrm{~m} /$ respective- 
ly during the control period) respectively reached $321.7 \mathrm{ml}(-17.2 \%)$ and $413.4 \mathrm{ml}(+7.4 \%)$ during the experimental period; ie, a $24.6 \%$ difference between these 2 extreme groups. These results could likely be explained by oxytocin levels reaching those obtained during milking and by the effect of milk transfer from the alveolar to the cisternal lumen. Additionally, progesterone could act directly on milk synthesis at the level of the secretory cells.

oxytocin / corpus luteum / milk ejection / galactopoiesis / ewe

\section{INTRODUCTION}

Àprès sa synthèse par les cellules épithéliales mammaires, le lait s'accumule dans la lumière des acini où il est momentanément stocké avant d'être transféré vers les régions proches du trayon par le réseau des canaux galactophores. Chez certaines espèces (rattes, lapine), ce transfert n'intervient que pendant la tétée, alors que chez d'autres, pourvues de volumineuses citernes (chèvres, brebis, vaches), il peut également avoir lieu entre les séquences d'allaitement ou de traite.

Chez la brebis, comme chez la plupart des femelles domestiques, l'ocytocine, qui est la principale hormone de l'éjection, ne provient pas exclusivement de la posthypophyse. En effet, ce nonapeptide peut aussi être synthétisé, stocké et libéré par le corps jaune (Wathes et Swann, 1982; Flint et Sheldrick, 1982 ; Watkins, 1983 ; Rodgers et al, 1983 ; Swann et al, 1984) et c'est ainsi que les augmentations de pression intramammaires observées dans cette espèce, après injection intrajugulaire de PGF2 $\alpha$ (Labussière et al, 1983), résultent probablement de l'action de cette hormone lutéale puisque les réponses sont abolies après castration (Labussière et al, 1986), lutectomie (Labussière et al, 1990) ou interruption de la circulation sanguine dans la vascularisation ovarienne (Labussière et Combaud, 1989).

Depuis Ely et Petersen (1941), it est bien admis qu'au moment de la traite ou de la tétée, l'éjection du lait dépend essen- tiellement de la libération d'ocytocine par le lobe nerveux de l'hypophyse à la suite de la stimulation des noyaux supra-optiques et para-ventriculaires de l'hypothalamus.

Par contre, on connaît beaucoup moins bien les mécanismes qui contrôlent entre les traites la descente du lait à proximité du trayon. L'ocytocine lutéale pourrait jouer à cet égard un rôle important et il est alors plausible d'admettre qu'un grand nombre de corps jaune puisse entraîner une ocytocinémie élevée et, par conséquent, une facilitation du transfert du lait alvéolaire vers les cavités citernales et une stagnation moins longue des produits de sécrétion dans la lumière des acini. II pourrait ainsi en résulter une stimulation de l'activité métabolique du parenchyme mammaire et une augmentation de la production laitière (Lakshmanan et al, 1958 ; Elliot, 1959 ; Linzell et Peaker, 1971 ; Labussière et al, 1978).

Ce sont ces hypothèses que nous avons voulu vérifier chez des brebis qui, dans un premier temps, sont dépourvues de corps jaune, puis, dans un deuxième temps, en possèdent un nombre variable à la suite de différents types de traitements superovulants.

\section{MATÉRIEL ET MÉTHODES}

\section{Présentation générale de l'essai}

La figure 1 schématise l'organisation générale de l'essai. Cinquante-neuf brebis adultes de 
race Lacaune, ayant mis bas entre le $1^{\mathrm{er}}$ janvier et le 15 février, sont laissées 48 heures avec leurs agneaux pour la tétée du colostrum. Elles sont alors définitivement séparées de ceux-ci et soumises 2 fois par jour à la traite mécanique ( $7 \mathrm{~h} 30$ et $16 \mathrm{~h}$ ).

L'expérience débute à la fin du $3^{e}$ mois postpartum à un stade où la production laitière régresse chaque jour régulièrement ; elle se subdivise en 2 périodes successives:

- la première (témoin) est caractérisée par l'absence de structure lutéale sur toutes les brebis. A cette fin, les corps jaunes éventuellement présents sont éliminés par 2 injections intramusculaires de $15 \mathrm{mg}$ de Dinolytic (Upjohn) à 10 jours d'intervalle et toute nouvelle ovulation est bloquée par une éponge de polyuréthane imprégnée de $30 \mathrm{mg}$ d'acétate de fluorogestone (FGA Intervet) placée pour 12 jours dans le vagin, 48 heures avant la seconde injection de Dinolytic ;

- la deuxième (expérimentale) est caractérisée par la présence d'un nombre variable de corps jaune (entre 0 et plus de 6) qui dépend du type de traitement appliqué au retrait de l'éponge (To) :

- soit renouvellement de l'éponge pour bloquer toute possibilité d'ovulation :
- soit injection intramusculaire de diverses doses de gonado-stimulines telles que:

- 300 ou 600 UI de PMSG à To ;

- $8 \mathrm{mg}$ de FSH en 2 administrations de $4 \mathrm{mg}$ à To et To $+12 h$;

- $16 \mathrm{mg}$ de FSH en 4 fractions de $6,5,3$ et $2 \mathrm{mg}$ respectivement aux temps To $-24 \mathrm{~h}$, To $12 \mathrm{~h}$, To et To $+12 \mathrm{~h}$.

Une laparatomie sous anesthésie générale (Labussière et al, 1986) réalisée en fin d'expérience (15 jours après To) permet de compter les corps jaunes et de classer a posteriori les brebis en 5 groupes (A, B, C, D, E) de 20, 7, 14, 7 et 11 animaux qui en possèdent respectivement $0,1,2,3$ à 6 et plus de 6 .

\section{Mesures du lait alvéolaire et citernal}

Le "transfert" de la fraction alvéolaire vers la citerne est apprécié en mesurant les volumes de la $1^{\text {re }}$ et de la $2^{\text {e }}$ émission lactée ( $V_{1}$ et $V_{2}$ ) sur les enregistrements des courbes de descente du lait obtenus selon la méthode déjà décrite par Labussière et Martinet (1964). Les mesures sont réalisées lors de 4 traites du soir consécutives, d'une part, au cours de la "première période témoin sans corps jaune" (5 à 8 jours

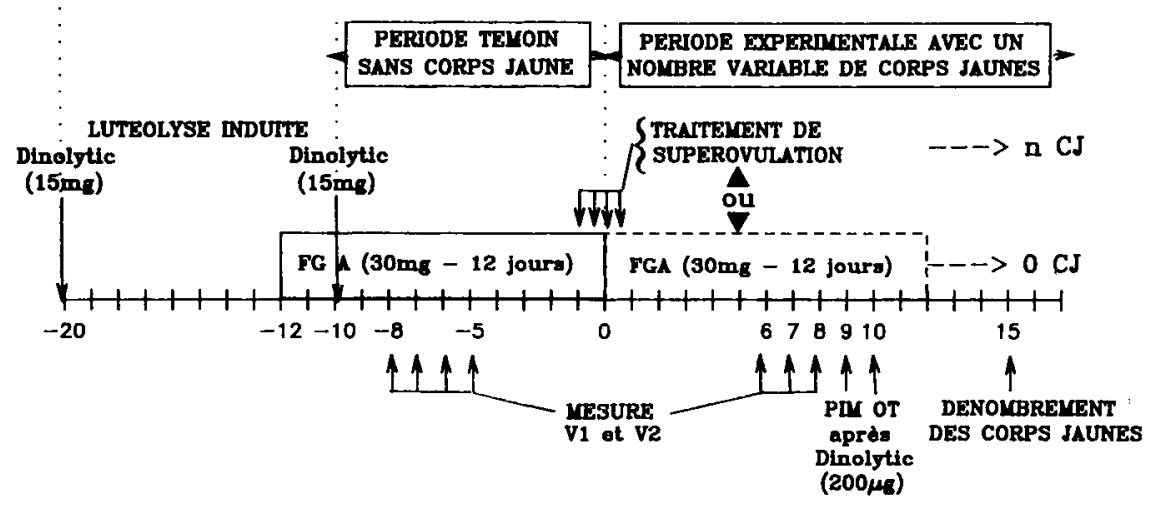

Fig 1. Plan de l'expérience. FGA: éponges de FGA posées durant 12 jours; $V 1$ : volume de lait citernal; V2: volume de lait alvéolaire; PIM et OT: pression intramammaire et ocytocinémie après injection de PGF2 $\alpha$. La progestérone et l'ocytocine plasmatique ont été mesurées chaque jour de J-2 à J17 (prélèvement de sang dans la veine jugulaire). 
avant le retrait de l'éponge) et, d'autre part, entre le $6^{\mathrm{e}}$ et le $8^{\mathrm{e}}$ jour de la "période expérimentale lutéale".

La production laitière totale recueillie à ces stades prend en compte non seulement le volume de ces 2 émissions mais aussi celui du lait recueilli à l'occasion de l'égouttage à la machine pratiqué juste avant l'enlèvement du faisceautrayeur.

\section{Contrôles de l'activité lutéale}

L'ocytocinémie et la progestéronémie "basales" sont estimées dans des échantillons de sang jugulaire recueillis quotidiennement à $7 \mathrm{~h} 30$ avant la traite du matin. Les premiers prélèvements sanguins débutent le lendemain du retrait de l'éponge ; les derniers ont lieu 17 jours plus tard.

En outre, les variations de la pression intramammaire et de l'ocytocinémie sont enregistrées respectivement les $9^{e}$ et $10^{e}$ jours après le retrait des éponges pour apprécier la réponse des brebis à l'injection intrajugulaire de $200 \mu \mathrm{g}$ d'un sel de trométhamine de PGF2 $\alpha$ (DinolyticUpjohn) ; ces mesures sont effectuées avant la traite du matin. Les échantillons sanguins destinés au dosage de l'ocytocine sont collectés dans la veine jugulaire 12, 7 et 2 minutes avant l'administration de PGF2 $\alpha$, puis $3,8,13,18$ et 28 minutes après celle-ci.

\section{Technique d'enregistrement de la pression intramammaire}

La traite du matin est retardée de 2 heures afin de réaliser les contrôles sur une mamelle bien remplie. Un cathéter en polyéthylène (type $P E$ 190 ; Intramédic, Clay Adams ; longueur : 100 $\mathrm{cm}$; diamètre extérieur $1,70 \mathrm{~mm}$; diamètre intérieur $1,19 \mathrm{~mm}$ ) est introduit (2 à $3 \mathrm{~cm}$ ) dans le canal du trayon ; l'autre extrémité de ce cathéter est reliée à un capteur de pression (Hewlett Packard type 1280 C) placé à hauteur de la citerne.

Les variations de pression intramammaire sont transcrites sur papier par l'intermédiaire d'un enregistreur Hewlett Packard (type 7754 A) équipé d'un préamplificateur Hewlett-Packard (modèle 8805 B).

\section{Dosages hormonaux}

Les échantillons sanguins sont prélevés grâce à des vacutainers de $10 \mathrm{ml}$ héparinés et prérérrigérés. Ceux-ci sont immédiatement centrifugés $\left(3000 \mathrm{G}, 2^{\circ} \mathrm{C}\right)$ pendant 15 minutes et le plasma est aussitôt placé au congélateur $\left(-20^{\circ} \mathrm{C}\right)$ jusqu'aux dosages radio-immunologiques de la progestérone (Saumande et al, 1985 ) et immunoenzymatique de l'ocytocine. Celui-ci est caractérisé par l'utilisation d'une phase de capture (IgG monoclonale de souris anti-lapin) et par le choix de l'acétylcholinestérase comme traceur enzymatique (Marnet et al, 1993). La sensibilité (ED 50\%), définie comme la quantité d'hormone qui entraîne un déplacement de $50 \%$ de l'activité totale introduite dans le dosage, est de $0,678 \pm 0,07 \mathrm{ng} / \mathrm{ml}$ pour la progestérone et de $23,1 \pm 0,53 \mathrm{pg} / \mathrm{ml}$ pour l'ocytocine.

\section{Analyses statistiques}

Pour chacun des groupes, l'évolution, entre période témoin et période expérimentale, des volumes moyens de lait citernal, de lait alvéolaire et de lait total a été soumise au test $t$ de Student par valeurs appariées.

Pour chacune des 2 périodes, les comparaisons entre groupes de ces mêmes critères ont été conduites :

- par le test de Scheffe destiné à déceler des différences de moyenne ;

- par le test non paramétrique de Jonckheere (1954) qui permet de révéler si le classement des moyennes de chaque lot est en relation avec celui du nombre de leurs corps jaunes.

\section{RÉSULTATS}

\section{Répartition du lait dans la mamelle et niveau de production}

Lorsqu'on compare les cinétiques d'émission du lait de la période expérimentale à celles situées 15 jours plus tôt pendant la période témoin, il apparaît (fig 2) que, plus le nombre de corps jaune est grand : 
- (i) plus le volume de lait citernal devient important. En effet, s'il diminue d'environ $10 \%$ chez les brebis du groupe A (qui n'ont pas de structures lutéales et qui, au-delà de 3 mois post-partum, sont naturellement soumises au ralentissement progressif de l'activité sécrétoire des cellules mammaires), il s'accroît par contre chez celles des groupes $B, C, D$ et $E$ qui ont formé respectivement 1,2,3 à 6 et plus de 6 corps jaunes. Selon le test non paramétrique de Jonckheere (1954), cet accroissement est très hautement significatif compte tenu de la valeur statistique calculée par cette méthode $(J=5,95)$ qui est très éloignée de zéro:

- (ii) plus le volume de lait alvéolaire est réduit. Le test de Jonckheere révèle que l'effet est également très hautement significatif $(\mathrm{J}=4,43)$ même si le classement des 5 groupes en fonction de l'ampleur de la réduction $(A, C, B, E, D)$ ne respecte pas strictement l'ordre (A, B, C, D, E) croissant des résultats de la superovulation ;

- (iii) plus le volume de lait total recueilli à la traite s'accroît (test de Jonckheere très hautement significatif ; $J=4,96$ ) et cet effet galactopoiétique est également mis en évidence au tableau I.

Ainsi pendant la période témoin, l'analyse de variance entre groupes indique que les 5 lots d'animaux ont le même niveau de production laitière alors que, pendant la période expérimentale, il se crée entre eux une différence significative. Dans ces conditions, le test de Scheffe révèle que les brebis sans corps jaune (groupe A) sécrètent alors significativement moins de lait que celles qui en possèdent plus de 6 (groupe $\mathrm{E}$ ).

La comparaison intragroupe (test $t$ de Student par valeurs appariées) indique qu'en 2 semaines le volume de lait du groupe $A$ baisse d'une façon très hautement significative de $388,6 \mathrm{ml}$ à $321,7 \mathrm{ml}$ $(-17,21 \%)$, ce qui reflète la diminution de la persistance de lactation, alors que celui du groupe $E$ progresse significativement de $384,8 \mathrm{ml}$ à $413,4 \mathrm{ml}(+7,43 \%)$. Entre ces 2 lots de brebis, ayant au préalable les mêmes potentialités laitières, il s'est

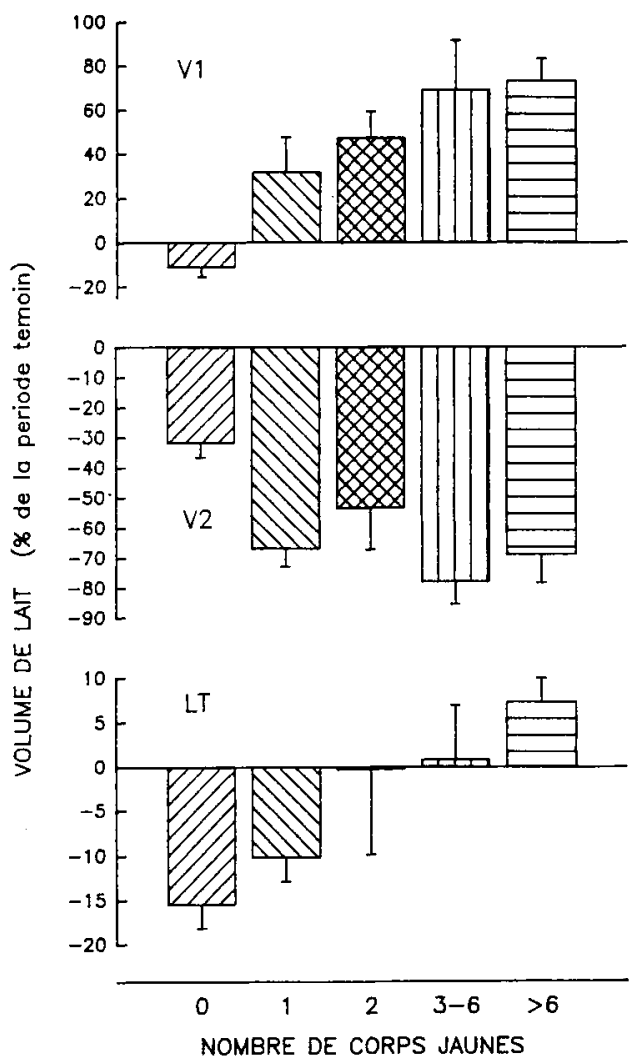

Fig 2. Modifications des volumes de lait citernal (V1), alvéolaire (V2) et de la production laitière totale (LT) en fonction du nombre de corps jaunes induits par les traitements de superovulation. Les modifications ( \pm SEM) sont celles de la période expérimentale exprimées en \% de la période témoin. Les valeurs $(\mathrm{J})$ fournies par le test de Jonckheere indiquent un effet très hautement significatif pour la diminution du lait alvéolaire $(\mathrm{J}=4,43$ ) et les augmentations du lait citernal ( $\mathrm{J}$ $=5,95)$ et du lait total $(\mathrm{J}=4,96)$. 


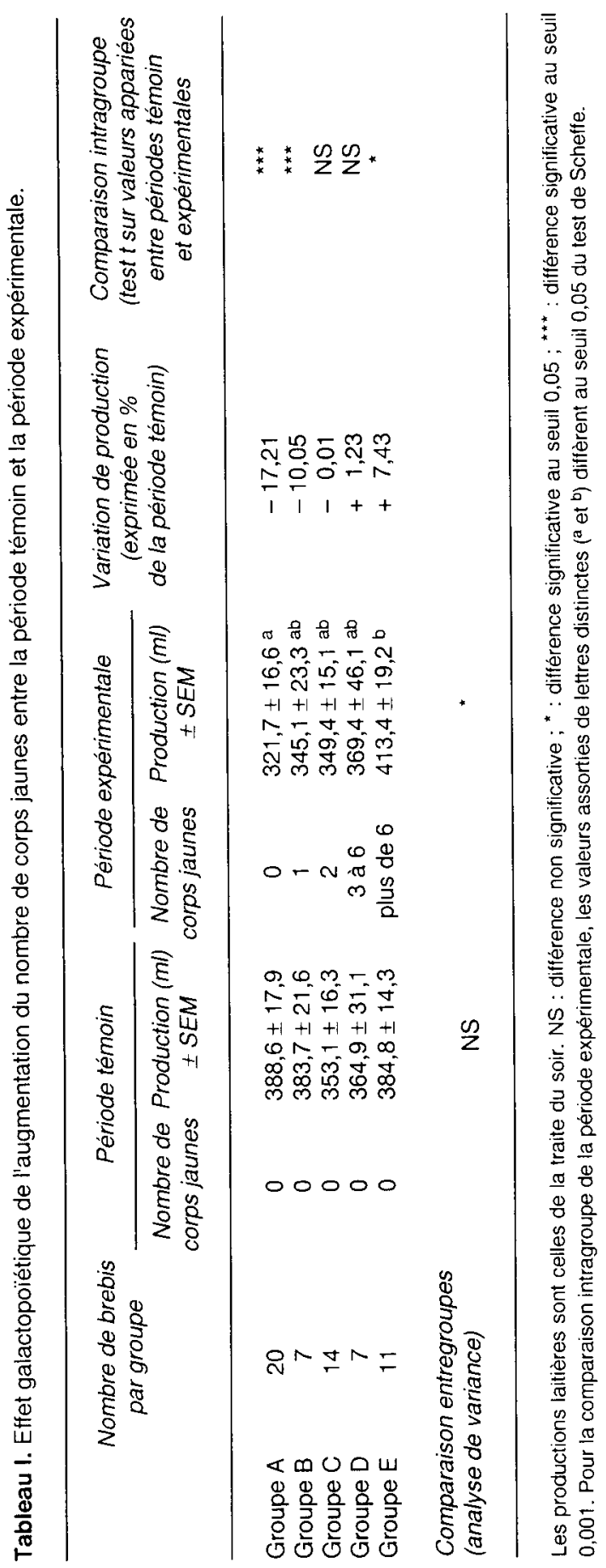


donc "creusé" en 15 jours un écart de $24,64 \%$.

Enfin le test $t$ par valeurs appariées montre également que le lait total du groupe $C$ n'a pas statistiquement varié entre la période témoin $(353,1 \mathrm{ml})$ et la période expérimentale $(349,6 \mathrm{ml})$; ceci suggère que la présence de 2 corps jaunes est capable de compenser l'affaiblissement
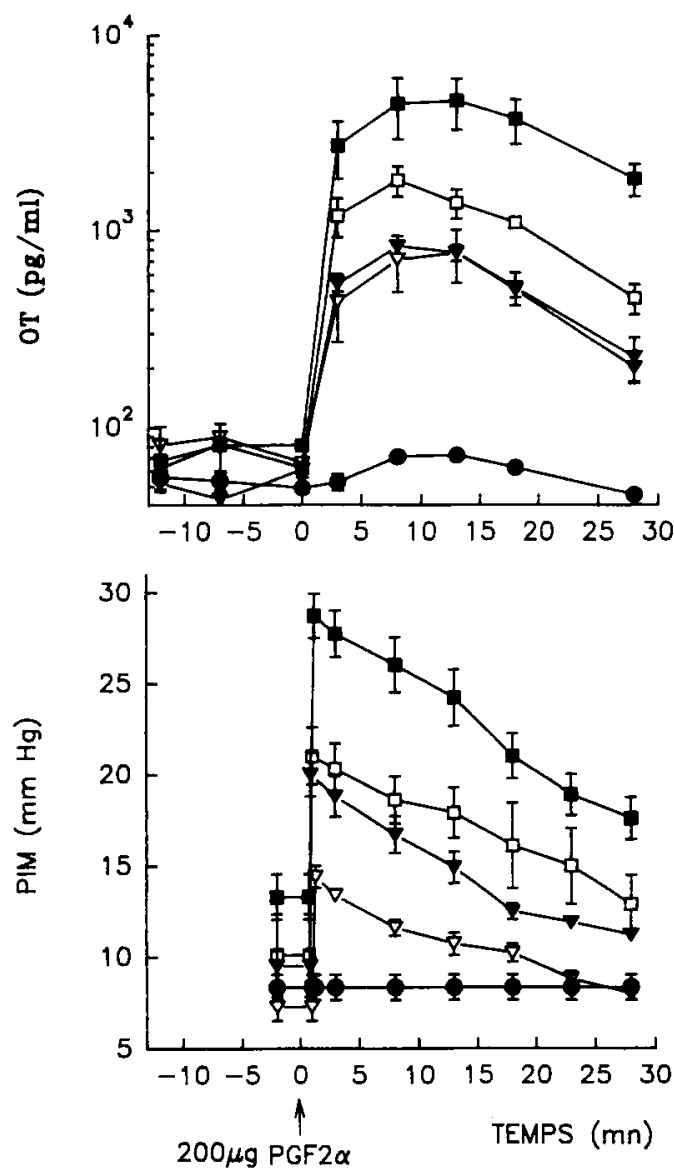

Fig 3. Cinétique ( \pm SEM) de l'ocytocinémie (OT) et de la pression intramammaire (PIM) après une injection de PGF2 $\alpha$ (Dinolytic $200 \mu \mathrm{g}$ IV) en fonction du nombre de corps jaunes induits par les traitements de superovulation. : lot $A, 0$ corps jaune; $\nabla:$ lot $B, 1$ corps jaune; $\nabla$ : lot $C$, 2 corps jaunes ; $\neg$ : lot $D, 3$ à 6 corps jaunes ; : lot $E$, plus de 6 corps jaunes. de l'activité sécrétoire des cellules mammaires pendant la phase décroissante de la courbe de lactation.

\section{Libération d'ocytocine et accroissement de la pression intramammaire après injection de PGF2 $\alpha$}

L'administration de $200 \mu \mathrm{g}$ de Dinolytic est suivie d'une augmentation de l'ocytocinémie et de la pression intramammaire (PIM) dont l'ampleur s'accroît avec le nombre de corps jaunes (fig 3). II faut également remarquer que la pression de base avant l'injection dépend déjà de ce nombre et que la latence entre l'injection de PGF2 $\alpha$ et le début de la réponse de PIM a alors tendance à diminuer $(58,8 \mathrm{~s} ; 50,3 \mathrm{~s} ; 46,9 \mathrm{~s}$ et 44,0 s) en fonction du nombre de corps jaunes observé (respectivement 1, 2, 3 à 6 et plus de 6).

\section{Évolution quotidienne de la progestéronémie et de l'ocytocinémie en l'absence d'injection de PGF2 $\alpha$}

II apparaît à la figure $\mathbf{4}$ que l'ocytocinémie mesurée dans la veine jugulaire sans injection préalable de PGF $2 \alpha$ (et avant la traite du matin pour réduire les libérations d'origine post-hypophysaire) et la progestéronémie sont d'autant plus élevées que le nombre de corps jaunes est important. Toutefois, en fin de phase lutéale les taux plasmatiques de progestérone semblent commencer à baisser plus précocement chez les femelles qui ont répondu intensément aux traitements de superovulation.

\section{DISCUSSION}

Cette étude montre qu'une augmentation du nombre de corps jaunes se traduit par: 
- (i) une facilitation entre les traites de la descente du lait alvéolaire vers les parties basses de la mamelle, proches du trayon. En effet, le volume du lait citernal (V1) s'accroît alors significativement tandis qu'en contre-partie le volume du lait présent dans la lumière des acini est significativement réduit. L'amélioration de l'efficacité de ce transfert est vraisemblablement la
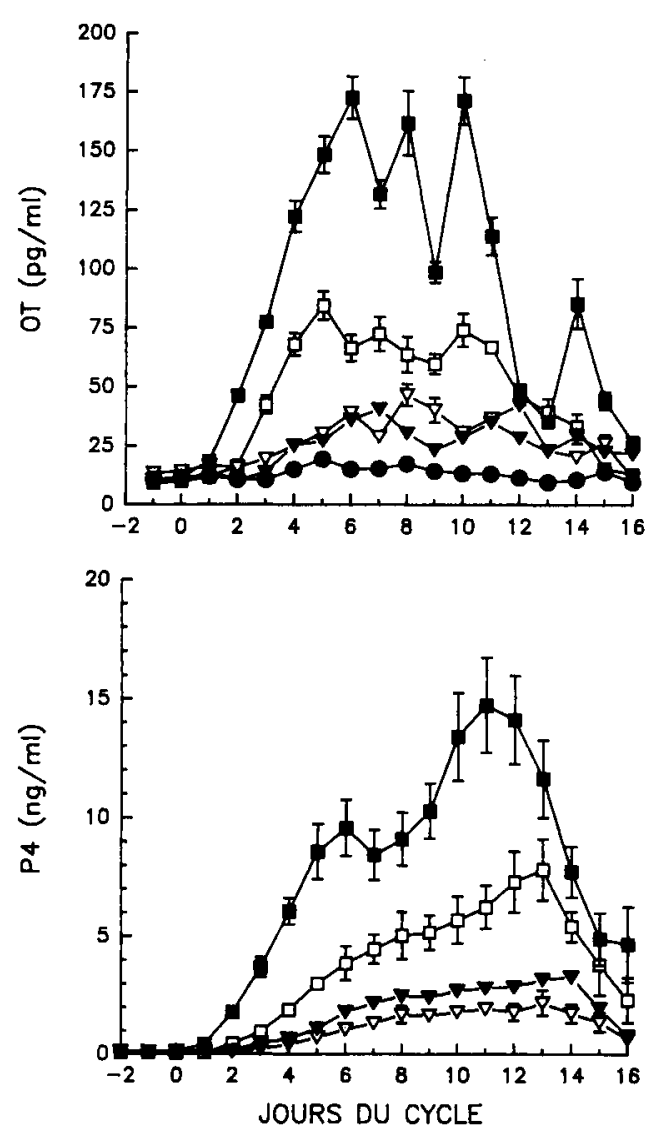

Fig 4. Niveaux plasmatiques de base ( \pm SEM) d'ocytocine (OT) et de progestérone (P4) en fonction du nombre de corps jaunes induits par les traitements de superovulation. : $\operatorname{lot} A, 0$ corps jaune; $\nabla$ : lot $B, 1$ corps jaune; $\nabla$ : lot $C, 2$ corps jaunes; $\square$ : lot $D, 3$ à 6 corps jaunes; $\mathbf{\square}$ : lot $E$, plus de 6 corps jaunes. conséquence de la forte ocytocinémie observée chez les brebis superovulées. Celles-ci présentent en effet une aptitude accrue à libérer l'ocytocine lutéale, non seulement après injection de $200 \mu \mathrm{g}$ de Dinolytic (comme l'indiquent les dosages plasmatiques et les enregistrements de pression intramammaire) mais aussi dans les conditions physiologiques du cycle en l'absence de toute administration de prostaglandines. Dans ce dernier cas, il est d'ailleurs intéressant de remarquer, d'une part, que l'ocytocinémie observée avec 3 corps jaunes (ou plus) est tout à fait comparable, voire supérieure, à celle obtenue au cours de la traite mécanique après la pose des gobelets (Mayer et al, 1989) et que, d'autre part, les taux circulants élevés sont atteints d'autant plus précocement que le nombre de corps jaune est important.

- (ii) un accroissement de la production laitière qui, par rapport aux brebis sans ovulation, peut atteindre $25 \%$ en 15 jours.

Cette importante stimulation galactopoïétique peut s'expliquer en partie par la facilitation de l'évacuation du lait hors de la lumière des acini; en effet, les incidences favorables d'une moindre stagnation lactée à proximité des cellules sécrétrices ont déjà été soulignées par plusieurs auteurs (Elliott, 1959; Morag, 1968) et notre équipe a pu démontrer l'influence bénéfique de la vacuité mammaire (Labussière et al, 1978) et de la plus grande fréquence des traites ou des tétées (Labussière et al, 1974).

Mais l'amélioration de l'activité métabolique des cellules mammaires chez les femelles polyovulées pourrait également être la conséquence de l'accroissement de la progestéronémie avec l'accroissement du nombre de corps jaunes. Cette hypothèse est étayée par nos observations récentes sur des brebis recevant différentes doses de progestagènes (Labussière et al, 1993) et qui manifestent une augmentation de 
production ; elle est conforme aux résultats de Barnawell (1967), de Ceriani (1970) ou de Smith et Inskeep (1970) mais contraire à ceux de Schilling et al (1980), de Troccon et al (1985), et de Cowen et Sosnik (1987). Des essais sont actuellement en cours pour la valider.

Il importe à cet égard de rappeler que l'imprégnation progestéronique intervient dans la régulation du nombre de récepteurs à l'ocytocine, mais les modalités de cette intervention semblent complexes. En effet, s'il est fréquemment admis que leur nombre sur l'utérus est plus fort en l'absence de progestérone (Nissenson et al, 1978 ; Soloff et al, 1983), il faut malgré tout signaler qu'un pré-traitement à l'acétate de fluorogestone suivi d'un traitement progestéronique peut conduire à des résultats inverses (Vallet et al, 1990) et il faut aussi savoir que les récepteurs à l'ocytocine de l'utérus et de la mamelle ne semblent pas être soumis au même type de régulation (Soloff et al, 1979; Soloff, 1982).

Ainsi, bien que nos 5 groupes d'animaux aient tous été soumis au même pré-traitement progestatif (FGA) de 12 jours pendant la période témoin, il n'est pas impossible que les différences de progestéronémie induite que nous avons constatées pendant la période expérimentale aient pu modifier la réceptivité à l'ocytocine des cellules myoépithéliales mammaires.

II apparaît donc souhaitable de poursuivre ces travaux sur l'effet galactopoïétique des corps jaunes multiples en cherchant à dissocier l'importance relative de l'ocytocinémie (qui facilite le transfert du lait alvéolaire dans la citerne) et de la progestéronémie qui pourrait intervenir non seulement sur le métabolisme des cellules sécrétrices mammaires mais aussi sur la réceptivité à l'ocytocine des cellules myoépithéliales.

\section{REMERCIEMENTS}

Ce travail a été réalisé grâce à l'efficace collaboration de Mme A Briand et de MM Chorho, G Guionneau, S Haigron, P Lambion, P Rolland et JB de Saint Jan, à qui nous adressons tous nos remerciements.

Nous exprimons également notre reconnaissance à M D André pour sa contribution aux dosages de progestérone plasmatique et à $M G$ Douaire pour ses conseils concernant l'interprétation statistique des résultats.

\section{RÉFÉRENCES}

Barnawell E (1967) Analysis of the direct action of prolactin and steroids on mammary tissue of the dog in organ culture. Endocrinology 80, 1083-1089

Ceriani RL (1970) Hormonal control of lactogenesis. J Dairy Res 38, 237-264

Cowen P, Sosnik U (1987) Effect of superovulation on lactating Holstein. Theriogenology 28 (6), 783-788

Elliott $G$ (1959) The direct effect of milk accumulation in the udder of the dairy cow upon milk secretion rate. Dairy Sci Abstr 21 (10), 435439

Ely E, Petersen WE (1941) Factors involved in the ejection of milk. J Dairy Sci 24, 211-223

Flint APF, Sheldrick EL (1982) Ovarian secretion of oxytocin in the sheep. J Physiol (Lond) $330,61 \mathrm{P}-62 \mathrm{P}$

Jonckheere AR (1954). A distribution free $k$-sample test against ordered alternatives. Biometrika 41, 133-145 (cité par Hollander M et Wolle DA, 1973. In: Non-parametric statistical methods, John Wiley and Sons, New York, 120-122)

Labussière J, Martinet J (1964) Description de deux appareils permettant le contrôle automatique des débits de lait au cours de la traite à la machine. Premiers résultats obtenus chez la brebis. Ann Zootech 13 (2), 199212

Labussière J, Combaud JF (1989) Effets des prostaglandines E1, E2, F1 $\alpha$ et F2 $\alpha$ sur l'éjection du lait de brebis soumises ou non au clampage de leur vascularisation ova- 
rienne. In: Proc 4th int symp machine milking of small ruminants ( $M$ Eitam ed) Ministère de l'Agriculture, Israël, 1-12

Labussière J, Combaud JF, Petrequin P (1974) Influence de la fréquence des traites et des tétées sur la production des brebis Préalpes du Sud. Ann Zootech 23 (4), 445-457

Labussière J, Combaud JF, Petrequin P (1978) Influence respective de la fréquence quotidienne des évacuations mammaires et des stimulations du pis sur l'entretien de la sécrétion lactée chez la brebis. Ann Zootech 27 (2), 127-137

Labussière $\mathrm{J}$, Philibert $\mathrm{C}$, Dotchewski $\mathrm{D}$, Combaud JF, de la Chevalerie FA, Bernabé J (1983) Effets des prostaglandines $F 2 \alpha$ sur l'éjection du lait de la brebis. Variations au cours du cycle sexuel. In: Proc IIth symp int ordeño mecanico de pequeños rumiantes. Sever Cuesta, Prado 10, Valladolid, Espagne, $64-80$

Labussière J, Eyi Ngui V, Combaud JF (1986) Effets des prostaglandines PGF2 $\alpha$ sur l'éjection du lait de la brebis. Conséquences de l'ovariectomie accompagnée ou non d'une complémentation oestroprogestative. Reprod Nutr Dev 26, 933-942

Labussière J, Lacroix MC, Combaud JF, de la Chevalerie FA, Thomas P (1990) Suppression par la lutectomie de l'accroissement simultané de la pression intramammaire et de l'ocytocinémie induit par l'injection de PGF $2 \alpha$ chez la brebis. Reprod Nutr Dev 30 , 91-96

Labussière J, Marnet PG, de la Chevalerie FA, Combaud JF (1993) Effets de la pose d'éponges vaginales (FGA) et de la superovulation induite par FSH et $\mathrm{LH}$ sur la sécrétion lactée et le volume des fractions citernales et alvéolaires des brebis Lacaune. In: Proc 5th int symp on machine milking of small ruminants (S Kukovics, ed) Budapest, 66-77

Lakshmanan S, Shaw JC, McDowell RE, Ellmore MF, Fohrman MH (1958) Short-interval milking as a physiological technique. 1. Effect of frequent milking with the aid of oxytocin on milk and milk fat production. J Dairy Sci 41, 1601-1608

Linzell JL, Peaker M (1971) The effects of oxytocin and milk removal on milk secretion in the goat. J Physiol 216, 717-734
Marnet PG, Volland H, Pradelles P, Grassi J, Beaufils $M$ (1993) Subpicogram determination of oxytocin by EIA technique using acetylcholinesterase as label. $J$ Immunoassay (sous presse)

Mayer H, Weber F, Segessemann V (1989) Oxytocin release and milking characteristics of Ostfriesian and Lacaune dairy sheep. In: Proc 4th int symp on machine milking of small ruminants ( $M$ Eitam, ed) Ministère de l'Agriculture, Israël, 548-563

Morag M (1968) The effect of varying the daily milking frequency on the milk yield of the ewe and evidence on the nature of the inhibition of milk ejection by half udder milking. Ann Zootech 17, 351-369

Nissenson R, Flouret G, Hechter O (1978) Opposing effect of estradiol and progesterone on oxytocin receptors in rabbit uterus. Proc Natl Acad Sci USA 75, 2044-2048

Rodgers RJ, O'Shea JD, Findlay JK, Flint APF, Sheldrick EL (1983) Large luteal cells, the source of luteal oxytocin in the sheep. Endocrinology 113, 2302-2304

Saumande J, Tamboura S, Chupin D (1985) Changes in milk and plasma concentrations of progesterone in cows after treatment to induce superovulation and their relationships with number of ovulations and of embryos collected. Theriogenology 23, 719-731

Schilling $E$, Smidt $D$, Sacher B, Schutzbar W (1980) Die Beeinträchtigung der Milchleistung durch die superovulation. Zuchtungskunde 52 (6), 421-428

Smith LW, Unskeep EK (1970) Effect of progestins on lactation in the ewe. J Anim Sci 30 (6) 957-959

Soloff MS (1982) Oxytocin receptors and mammary myoepithelial cells. J Dairy Sci 65, 326337

Soloff MS, Alexandrova M, Fernstrom M (1979) Oxytocin receptors: triggers for parturition and lactation? Science 204, 1313-1314

Soloff MS, Fernstrom MA, Periyasamy S, Soloff S, Baldwin S (1983) Regulation of oxytocin receptor concentration in rat uterine explants by estrogen and progesterone. Can J Biochem Cell Bio/ 61, 625-630

Swann RW, O'Shaughnessy PJ, Birkett SD, Wathes DC, Porter DG, Pickering BT (1984) 
Biosynthesis of oxytocin in the corpus luteum. Febs Lett 174, 262-266

Troccon JL, Ozil JP, Chupin D (1985) Production par transplantation embryonnaire, de veaux monozygotes. Aspects zootechniques. Bull Tech CRVZ INRA Theix 59, 19-28

Vallet JL, Lamming GE, Batten M (1990) Control of endometrial oxytocin receptor and uterine response to oxytocin by progesterone and oestradiol in the ewe. J Reprod Fertil 90, 625-634

Wathes DC, Swann RW (1982) Is oxytocin an ovarian hormone? Nature (Lond) 297, 225-227

Watkins WB (1983) Immunohistochemical localization of neurophysin and oxytocin in the sheep corpora lutea. Neuropeptides 4, 51-54 